\title{
Strates
}

STRATES Matériaux pour la recherche en sciences sociales

Hors-série | 2002

Parcours dans la recherche urbaine, Michel Rochefort, un géographe engagé

\section{Une étonnante réunion à la Datar}

\section{Pierre Beckouche}

\section{(2) OpenEdition \\ Journals}

Édition électronique

URL : http://journals.openedition.org/strates/517

DOI : $10.4000 /$ strates.517

ISSN : $1777-5442$

Éditeur

Laboratoire Ladyss

Édition imprimée

Date de publication : 1 janvier 2002

ISSN : 0768-8067

Référence électronique

Pierre Beckouche, «Une étonnante réunion à la Datar », Strates [En ligne], Hors-série | 2002, mis en ligne le 02 mai 2005, consulté le 08 septembre 2020. URL : http://journals.openedition.org/strates/ 517 ; DOI : https://doi.org/10.4000/strates.517

Ce document a été généré automatiquement le 8 septembre 2020

Tous droits réservés 


\title{
Une étonnante réunion à la Datar
}

\author{
Pierre Beckouche
}

1 J'ai récemment assisté avec quelques collègues à une étonnante réunion à la Datar. Son objet était de faire réagir les universitaires à la nouvelle organisation administrative du territoire telle qu'elle s'esquisse avec les projets de loi Voynet et Chevènement.

2 Finis la dilution communale et l'égoïsme municipal, nous aurons des ensembles intercommunaux (à défaut de structures supracommunales qui viendront peut-être un jour), les «Agglomérations » pour les grandes villes, les «Pays» ailleurs. Dans les Agglomérations un établissement public de coopération inter-communale prélèvera la taxe professionnelle. Des contrats d'Agglomération passés avec l'État à partir de projets proposés par les acteurs locaux, seront favorisés par une carotte financière pas encore fixée mais qu'on espère significative pour donner corps à cette philosophie des «territoires de projet». Le projet de loi projette de se donner du temps pour que ces projets de territoires aboutissent à des territoires de projet, pour certaines Agglomérations très vite, pour d'autres pas avant cinq ou dix ans, pour d'autres plus tard encore. Limpide, non?

3 Comme rien n'est simple, les contrats de Ville, qui sont la forme nouvelle de contractualisation de la politique de la Ville, constitueront le volet social des futurs contrats d'Agglomération. Or les contrats de Ville, qui bénéficient de l'expérience déjà ancienne de la politique de la Ville et doivent répondre à l'urgence sociale, devront, eux, être ficelés le 1er janvier 2000 pour être intégrés aux prochains contrats de plan État-région.

4 Car c'est là que les choses deviennent amusantes. Les prochains contrats de plan comprendront un volet territorial, doté d'une partie des montants du contrat, pour mieux ancrer les projets d'investissement public sur des réalités territoriales de plus en plus différenciées. Un volet territorial dit externe devra donner une vision d'ensemble des enjeux de la région; un volet interne déterminera des sous-ensembles posant des problèmes spécifiques, auxquels des politiques publiques mieux coordonnées devront répondre. Donc, si l'on me suit bien, les contrats de Ville devront se caler dans cette déclinaison infrarégionale des prochains contrats de plan. 
5 Évidemment, une petite question demeure : comment dessiner ces sous-ensembles régionaux? La question n'est pas des plus faciles, d'autant qu'il faudra tenir compte d'un nouvel impératif issu du rapport Auroux : la simplification des zonages des politiques territoriales françaises et leur adéquation avec ceux de la politique régionale de la DG XVI (Direction générale de la Commission européenne chargée des politiques régionales). Comme on sait, les financements d'un contrat de plan sont abondés par l'État, les collectivités locales, et les Fonds structurels. Désormais, la carte des zones aidées par les Fonds structurels influencera celle de la PAT $^{1}$ et autres fonds de l'aménagement du territoire. Il est donc probable que le dessin des sous-ensembles d'une région tiendra compte de cette nouvelle grammaire géographico-financière.

6 Je passe sous silence le petit détail de la réforme des Fonds structurels : le passage de six objectifs à trois, la réduction de la part de la population couverte par les Fonds structurels, vont entraîner une refonte du zonage de Bruxelles. Mais enfin, tout devrait être parfaitement clair d'ici quelques temps, n'en doutons pas.

7 En revanche, la question de la détermination de sous-ensembles régionaux restera infernale dans le cas francilien: comment découper une aire métropolitaine, précisément caractérisée par l'interdépendance de ses territoires locaux ? Nul doute que l'administration, assistée par de brillants universitaires, parviendra à réduire la difficulté.

8 Je veux bien reconnaître que cette architecture virtuelle n'apparaît pas dans une totale clarté. Pourtant je n'en dirai pas plus. D'abord parce que je ne suis pas sûr d'en comprendre tout le sel, et surtout parce que les débats parlementaires qui viennent risquent suffisamment de changer les textes pour qu'on s'en tienne à un prudent attentisme. Par exemple, le projet concomitant de réforme de la taxe professionnelle proposé par Bercy, qui prévoit de supprimer la partie salaires de l'assiette, risque de vider de la moitié de sa substance l'objectif de mise en commun intercommunale des recettes. Mais nous n'en sommes pas à ça près... Seule certitude, le rôle de la Datar paraît renforcé : elle seule peut maîtriser cette ingénierie institutionnelle démoniaque, l'articulation des échelles, l'articulation interministérielle des politiques sectorielles de l'État appliquée à des territoires infrarégionaux, et l'articulation des différentes formes de contractualisation.

9 En réalité, après l'exposé du contexte politico-institutionnel, notre réunion prit un tour inattendu : les fonctionnaires de la Datar se tournèrent vers les universitaires pour leur avouer l'étendue de leurs doutes et de leurs questions. La séance se termina par un brain storming débridé et passionnant, mais qui laisse songeur quand on pense à la maîtrise requise par les transformations institutionnelles promises.

10 Ce désarroi n'a, à la réflexion, rien de surprenant. La Datar est face à une quadruple crise. Crise des résultats, d'abord : le rééquilibrage Paris-province a beaucoup progressé avec la déconcentration et la décentralisation, avec le développement d'équipements structurants dans toutes les villes et régions de province. Mais d'un autre côté, Paris reste la seule ville de dimension vraiment internationale, l'élite sociale et économique demeure aussi parisienne qu'il y a trente ans. Par ailleurs le déclin des régions de tradition industrielle, à commencer par le Nord-Pas-de-Calais, n'est pas enrayé.

11 Crise des objectifs : faut-il continuer à viser l'égalité territoriale, ou au contraire conforter les zones les plus productives qui sont aussi les meilleures armes françaises dans la compétition internationale? Le discours de la Datar sur la région parisienne a 
changé depuis quelques années. Ce n'est plus : le problème français, c'est que Paris est trop gros et étouffe la province; la nouvelle doctrine (personnellement j'y souscris) est : il n'y a pas assez de régions parisiennes en province, c'est-à-dire d'alternatives métropolitaines à Paris. L'aménagement du territoire, c'est l'arbitrage entre équité spatiale et efficacité économique ; en ces temps de compétition économique à tout crin, l'arbitrage penche plus que naguère vers l'efficacité.

Un autre objectif classique bat de l'aile: celui de la réduction des inégalités interrégionales. Non qu'elles aient disparu, bien entendu. Mais elles sont beaucoup moins grandes qu'on le croit souvent - surtout si l'on s'intéresse aux revenus par habitant et pas au PIB par habitant, car les prélèvements obligatoires sont une gigantesque machine à redistribuer de la richesse, socialement et géographiquement. Surtout, les principales disparités et les principaux enjeux territoriaux se situent désormais à une échelle intrarégionale, et notamment au sein de l'espace urbain.

Enfin l'objectif de réduire la fracture sociale en s'attaquant aux inégalités spatiales ne résiste pas à un examen sérieux. La confusion est pourtant entretenue par la DG XVI, pour laquelle le Traité de Maastricht a fait « de la cohésion sociale et du même coup de l'équilibre entre les régions un objectif majeur de l'Union à côté de la réalisation du marché intérieur et de l'UEM ${ }^{2} »$ (Europe 2000 +). En France, le (pseudo) grand débat qui avait préparé la $\mathrm{LOADT}^{3}$ de 1995 avait eu un large recours à ce glissement spécieux. Mais je crois que l'opération qui consiste à traiter les problèmes spatiaux à la place des problèmes sociaux ne convainc plus beaucoup. La politique de la Ville elle-même renonce à se focaliser sur quelques quartiers. Le rapport Cavallier pointe bien la diversité des mécanismes institutionnels et sociaux qui concourent à la ségrégation socio-spatiale, il souligne justement la nécessité de les prendre en compte à l'échelle de l'ensemble de l'agglomération.

14 Crise des acteurs : la Datar a eu longtemps le monopole du discours sur le territoire national, elle ne l'a plus. De nouveaux acteurs ont émergé. Les collectivités locales d'abord ; le rôle croissant des contrats de plan comme cadre de l'action publique, ainsi que le projet de Loi Voynet ont confirmé le rôle des régions dans l'aménagement du territoire - on devrait plutôt dire des territoires. Les grandes entreprises privées de réseaux ensuite, qui produisent de l'espace sur-mesure. Bruxelles enfin, dont les Fonds structurels destinés à l'hexagone valent huit fois le budget de la Datar (16 milliards par an contre moins de deux, sans compter les 50 ou 60 milliards de la Politique agricole commune).

Crise des moyens pour terminer: on ne sait plus exactement comment aider les territoires en difficulté. Faut-il des primes à l'implantation d'entreprise, dont l'efficacité est douteuse et le principe combattu par la DG XI (Direction générale de la Commission européenne chargée de l'environnement)? Faut-il financer des infrastructures, dont l'écheveau est maintenant largement tissé sur l'ensemble de l'Hexagone? L'échec de l'aide pour le Mezzogiorno en Italie et celui, plus récent, pour les Länder de l'ex-RDA (plus de mille milliards de francs transférés d'Allemagne de l'Ouest en Allemagne de l'Est depuis 1990) jettent un trouble sur la politique régionale dans son ensemble.

D'où le remue-méninges à la Datar. D'où aussi le vif intérêt que l'institution trouve dans ce projet d'architecture du territoire national en Agglomérations. Elles seront au nombre d'une centaine (le projet de loi proposait de prendre les villes de plus de 50000 habitants, les premiers débats parlementaires ont relevé le seuil pour ne pas laisser aux 
Pays la portion congrue). Cet objet géographique présente le grand avantage d'avoir une double signification territoriale: il désigne à la fois les principales villes de l'armature urbaine, donc de l'organisation inter-régionale de l'espace national - le métier de base de la Datar. Il désigne aussi les villes au sein desquelles sont posés l'essentiel des problèmes territoriaux d'aujourd'hui: ségrégation socio-spatiale, périurbanisation et consommation d'espace, système de transport et questions environnementales, etc. Bref, les Agglomérations sont pour la Délégation un enjeu de connaissance et d'action dans lequel, l'avenir nous le dira peut-être, elle joue sa crédibilité voire sa survie.

Par tradition et par culture, la Datar connaît mieux l'inter-régional que l'urbain. Voilà pourquoi les universitaires de notre réunion étaient plutôt des spécialistes de l'urbain. La demande était franche: organisez-nous un transfert de connaissances sur les territoires urbains. Quant à l'aspect armature urbaine de nos Agglomérations, il ne faut pas croire que l'assurance de la Datar soit tellement plus établie : il faut promouvoir des alternatives métropolitaines à Paris, en faveur de villes qui ont d'ores et déjà une capacité d'entraînement sur leur environnement régional et peuvent prétendre à un rang européen. Soit, mais plus précisément? Dans quelles directions orienter les recherches? Notre brain storming n'a pas débouché sur des innovations radicales. Je dois à la vérité dire que nous avons terminé la réunion en évoquant... les métropoles d'équilibre et les travaux de Michel Rochefort.

\section{NOTES}

1.Politique d'Aménagement du Territoire.

2.Union Économique et Monétaire.

3.Loi d'Orientation pour l'Aménagement du Territoire.

\section{AUTEUR}

\section{PIERRE BECKOUCHE}

Professeur de géographie à l'université de Paris I 